\title{
An Error Analysis for the Finite Element Approximation to the Steady-State Poisson-Nernst-Planck Equations
}

\author{
Ying Yang ${ }^{1, *}$ and Benzhuo $\mathrm{Lu}^{2}$ \\ ${ }^{1}$ Department of Computational Science and Mathematics, Guilin University of \\ Electronic Technology, Guilin 541004, Guangxi, China \\ ${ }^{2}$ LSEC, Institute of Computational Mathematics and Scientific/Engineering Computing, \\ the National Center for Mathematics and Interdisciplinary Sciences, Academy of \\ Mathematics and Systems Science, Chinese Academy of Sciences, Beijing 100190, China
}

Received 2 December 2011; Accepted (in revised version) 26 July 2012

Available online 25 January 2013

\begin{abstract}
Poisson-Nernst-Planck equations are a coupled system of nonlinear partial differential equations consisting of the Nernst-Planck equation and the electrostatic Poisson equation with delta distribution sources, which describe the electrodiffusion of ions in a solvated biomolecular system. In this paper, some error bounds for a piecewise finite element approximation to this problem are derived. Several numerical examples including biomolecular problems are shown to support our analysis.
\end{abstract}

AMS subject classifications: $65 \mathrm{~N} 30,92 \mathrm{C} 40$

Key words: Poisson-Nernst-Planck equations, finite element method, error bounds.

\section{Introduction}

In this paper, we shall analyze the finite element approximation for a widely used mathematical model-Poisson-Nernst-Planck (PNP) equations in the biomolecular modeling area as follows:

$$
\begin{cases}\nabla \cdot D^{i}\left(\nabla p^{i}+\beta q^{i} p^{i} \nabla \phi\right)=0, & \text { in } \Omega_{s}, 1 \leq i \leq n, \\ -\nabla \cdot(\epsilon \nabla \phi)-\lambda \sum_{i=1}^{n} q^{i} p^{i}=\rho^{f}, & \text { in } \Omega,\end{cases}
$$

where $\phi$ is the electrostatic potential and $p^{i}$ is the concentration of the $i$-th ion species. This model is used to describe the electrodiffusion of mobile ions in a solvated biomolecular system. The model and its application have been discussed by authors such

*Corresponding author.

Email: yangying@1sec.cc.ac.cn (Y. Yang), bzlu@lsec.cc.ac.cn (B. Z. Lu) 


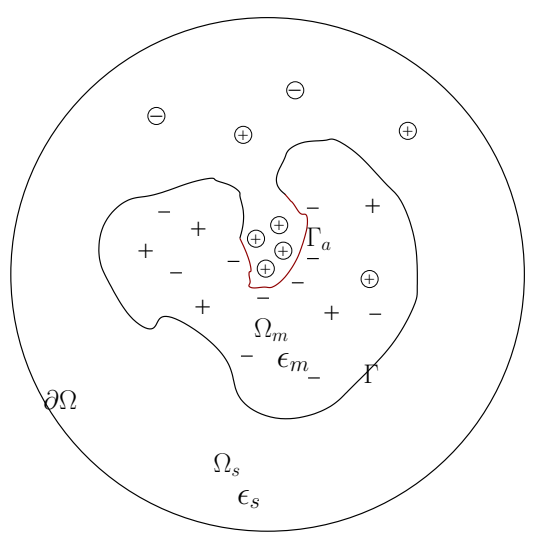

Figure 1: 2-D illustration of the computational domain modeling a solvated biomolecular system.

as $[14,16,17]$. Here the electrostatic potential is induced by the mobile ions and the fixed charges carried by biomolecules. Fig. 1 illustrates a solvated biomolecular system in an open domain $\Omega \subset \mathbb{R}^{3}$. The domain $\Omega_{m} \subset \Omega$ represents the biomolecule(s) and the remain domain $\Omega_{s}=\Omega \backslash \bar{\Omega}_{m}$ shows a solvent surrounding the biomolecule(s). The molecular surface $\Gamma$ interfaces domains $\Omega_{m}$ and $\Omega_{s}$. Charged ligands in this model are also treated as diffusive species, and might react with the biomolecules on a part of the molecular surface $\Gamma_{a}$. The dielectric $\epsilon$ is a piecewise constant and the permanent (fixed) charge distribution $\rho^{f}=\sum_{j=1}^{N_{m}} q_{j} \delta\left(x-x_{j}\right)$ is a combination of Dirac distributions at singular points $x_{j}$, $j=1,2, \cdots N_{m}$. The diffusive particles are distributed in $\Omega_{s}$.

Since the analytic solutions of the PNP equations only exist in very few cases for simple shape molecules with continuous dielectric coefficient $\epsilon$, numerical solutions of the PNP equations become natural. A variety of numerical methods such as finite element method, finite difference method and boundary element method etc. have been applied to solving the PNP and PNP-like systems (cf. $[6,9,10,13-15,19,20,27]$ etc). Among those approaches, the finite element method is considered to be very promising in which irregular shapes can be fitted more easily. Moreover, the finite element method allows fine meshes to be put where they are needed, such as at interfaces, and coarser meshes to be put far from the molecule, where spatial changes in electrostatic potential are small.

In contrast to amount of work on the numerical computations of PNP equations, the work of mathematical analysis of PNP equations seems very limited, especially for finite element method. The existence and stability for the solutions of PNP equations are established by [12] for 1-D PNP model for electron flows in semiconductors. Singular perturbation methods and asymptotic analysis are applied to study the solution properties of 1-D PNP equations in [5]. We are not aware of any mathematical analysis for the finite element approximation of the PNP equations.

The main difficulties for mathematical analysis for the PNP equations (1.1) are Dirac distribution sources and nonlinear coupling. To deal with the Dirac distribution sources, 
we decompose the electrostatic solution $\phi$ as an unknown solution $\tilde{\phi} \in H^{1}$ of a regularized Poisson equation and a known Green function $G$ as follows (see Section 3 below):

$$
\phi=\tilde{\phi}+G \text {. }
$$

Such a decomposition is commonly used in numerical computation (see e.g., $[14,28]$ ) and mathematical analysis (see e.g., [7,22]) of the equations with Dirac distribution sources. In [7], the error bounds for the finite element approximation to the regularized solution $\tilde{\phi}$ are given for the Poisson-Boltzmann equation which is not a coupled system. To deal with the nonlinear coupling, our arguments here build upon the techniques of [11]. In [11], the error bounds for the Joule heating problem are presented (some related works on numerical analysis for such kind of nonlinear problems refer to e.g., [2,24]). The Joule heating problem is also a coupled system, but it contains no Dirac distribution sources. In addition, the PNP equations couple only in a local domain, which is different from the Joule heating problem and brings more difficulties to get the error bounds, for example, we have to deal with some local error estimates.

Next, let us give a somewhat more detailed but informal description of the main result. Let $\left(\phi_{h}, p_{h}^{i}\right)$ be the linear finite element approximation to the solution of problem (1.1). We first present some local error bounds for $\phi_{h}$ as follows:

$$
\left\|\phi-\phi_{h}\right\|_{0, \Omega_{0}} \leq C\left(h^{\frac{3}{2}}|\ln h|^{\frac{3}{2}}+\sum_{i=1}^{n}\left\|p^{i}-p_{h}^{i}\right\|_{0, \Omega_{s}}\right)
$$

and

$$
\left\|\phi-\phi_{h}\right\|_{1, \Omega_{s}} \leq C\left(h^{s}+\sum_{i=1}^{n}\left\|p^{i}-p_{h}^{i}\right\|_{0, \Omega_{0}}\right), \quad 0<s \leq 1
$$

where $\Omega_{0} \subset \subset \Omega$ and all the singular points $x_{j} \notin \Omega_{0}$. Then the error bounds for $p_{h}^{i}$ are given as follows: for any $v_{h} \in S_{0}^{h}\left(\Omega_{s}\right)$ with $v_{h}=0$ on $\partial \Omega$, we have

$$
\left\|p^{i}-p_{h}^{i}\right\|_{1, \Omega_{s}} \leq C\left(\left(h^{s}+\sum_{i=1}^{n}\left\|p^{i}-p_{h}^{i}\right\|_{0, \Omega_{s}}\right)\left(1+h^{-\frac{3}{2}}\left\|p^{i}-p_{h}^{i}\right\|_{0, \Omega_{s}}\right)+\left\|p^{i}-v_{h}\right\|_{1, \Omega_{s}}\right), \frac{1}{2}<s \leq 1
$$

and if $p_{h}^{i} \in L^{\infty}\left(\Omega_{s}\right)$, then

$$
\left\|p^{i}-p_{h}^{i}\right\|_{1, \Omega_{s}} \leq C\left(h^{s}+\sum_{i=1}^{n}\left\|p^{i}-p_{h}^{i}\right\|_{0, \Omega_{s}}+\left\|p^{i}-v_{h}\right\|_{1, \Omega_{s}}\right), \quad 0<s \leq 1 .
$$

The error bound for $\phi-\phi_{h}$ on the domain $\Omega$ is also given as follows:

$$
\left\|\phi-\phi_{h}\right\|_{0, \Omega} \leq C\left((1+|\ln h|) h^{\frac{1}{2}}+\sum_{i=1}^{n}\left\|p^{i}-p_{h}^{i}\right\|_{0, \Omega_{s}}\right) .
$$


All the error bounds above are obtained under the assumption

$$
\phi \in W^{1, \infty}\left(\Omega_{s}\right) \cap H^{1+s}\left(\Omega_{s}\right) \cap W_{0}^{1, p}(\Omega), \quad 0<s \leq 1, \quad 1 \leq p \leq \frac{3}{2}
$$

and

$$
p^{i} \in W^{1+s, \infty}\left(\Omega_{s}\right), \quad 0<s \leq 1 .
$$

This assumption is essentially the same as in the standard error analysis for the corresponding linear elliptic problems with Dirac distribution sources (cf. [23]). In addition, some numerical examples including biomolecular problems are shown to verify the theoretic results.

The paper is organized as follows. In the following section, some preliminaries are presented. In Section 3, the error bounds for the finite element approximation are given. Some numerical examples are shown in Section 4.

\section{Preliminaries}

In this section, we shall first describe some basic notation, then introduce the steadystate Poisson-Nernst-Planck equations and boundary conditions. Some properties on the finite element approximations are also studied in this section. Let $\Omega \subset \mathbb{R}^{3}$ be a polyhedral convex domain with a Lipschitz-continuous boundary $\partial \Omega$. We shall adopt the standard notation for Sobolev spaces $W^{s, p}(\Omega)$ and their associated norms and seminorms, see, e.g., [1,3]. For $p=2$, we denote $H^{s}(\Omega)=W^{s, 2}(\Omega)$ and $H_{0}^{1}(\Omega)=\left\{v \in H^{1}(\Omega):\left.v\right|_{\partial \Omega}=0\right\}$, where $\left.v\right|_{\partial \Omega}=0$ is in the sense of trace, $\|\cdot\|_{s, p, \Omega}=\|\cdot\|_{W^{s, p}(\Omega)}$ and $(\cdot, \cdot)$ is the standard $L^{2}$ inner product. Assume that $T^{h}(\Omega)$ is a quasi-uniform mesh of size $h \in\left(0, h_{0}\right)$ for some fixed $h_{0}>0$. We define linear finite element spaces

$$
S^{h}(\Omega)=\left\{v \subset H^{1}(\Omega):\left.v\right|_{e} \in P^{1}(e), \forall e \in T^{h}(\Omega)\right\}, \quad S_{0}^{h}(\Omega)=S^{h}(\Omega) \cap H_{0}^{1}(\Omega),
$$

where $P^{1}(e)$ is the set of linear polynomials. Define

$$
S_{h}^{0}(G)=\left\{v \mid v \in S^{h}(G), \operatorname{supp} v \subset \subset G\right\} .
$$

For $D \subset G \subset \Omega$, we write $D \subset \subset G$ to mean that $\operatorname{dist}(\partial D \backslash \partial \Omega, \partial G \backslash \partial \Omega)>0$.

In this paper, we consider the following steady-state Poisson-Nernst-Planck system for simulating biomolecular diffusion-reaction process (cf. [14])

$$
\begin{cases}\nabla \cdot D^{i}\left(\nabla p^{i}+\beta q^{i} p^{i} \nabla \phi\right)=0, & \text { in } \Omega_{s}, 1 \leq i \leq n, \\ -\nabla \cdot(\epsilon \nabla \phi)-\lambda \sum_{i=1}^{n} q^{i} p^{i}=\rho^{f}, & \text { in } \Omega \subset R^{3}\end{cases}
$$


with the following interface conditions and boundary conditions (for simplicity, the reactive molecular surface $\Gamma_{a}$ is not considered)

$$
\begin{cases}{[\phi]_{\Gamma}=0, \quad\left[\epsilon \frac{\partial \phi}{\partial n}\right]_{\Gamma}=0,} & \text { on } \Gamma=\bar{\Omega}_{s} \cap \bar{\Omega}_{m} \\ n \cdot D^{i}\left(\nabla p^{i}+\beta q^{i} p^{i} \nabla \phi\right)=0, & \text { on } \Gamma, 1 \leq i \leq n, \\ \phi=0, & \text { on } \partial \Omega, \\ p^{i}=p_{\text {pulk }}^{i} & \text { on } \partial \Omega,\end{cases}
$$

where $p^{i}(x)$ is the concentration of the $i$-th species particle carrying charge $q^{i}, \phi(x)$ is the electrostatic potential, $D^{i}(x)$ is the diffusion coefficient, $\rho^{f}(x)=\sum_{j=1}^{N_{m}} q_{j} \delta\left(x-x_{j}\right)$ is an ensemble of singular charges $q_{j}$ located at $x_{j}$ inside biomolecules, $\beta=1 /\left(\kappa_{B} T\right)$ is the inverse Boltzmann energy,

$$
\epsilon(x)= \begin{cases}\epsilon_{m}, & x \in \Omega_{m} \\ \epsilon_{s}, & x \in \Omega_{s}\end{cases}
$$

is the dielectric coefficient,

$$
\lambda= \begin{cases}0, & \text { in } \Omega_{m} \\ 1, & \text { in } \Omega_{s},\end{cases}
$$

and $p_{\text {pulk }}^{i}, i=1,2 \cdots, n$ are given functions. We will assume the interface $\Gamma$ to be sufficiently smooth, say, of class $C^{2}$.

The week formulations of (2.1) and (2.2) are that: find $\phi \in W_{0}^{1, p}(\Omega) \cap H^{1}\left(\Omega_{s}\right)(1 \leq p \leq$ $3 / 2)$ and $p^{i} \in V=\left\{v\left|v \in H^{1}\left(\Omega_{s}\right), v\right|_{\partial \Omega}=p_{p u l k}^{i}\right\}(1 \leq i \leq n)$ such that

$$
\begin{array}{ll}
\left(D^{i} \nabla p^{i}, \nabla v\right)+\left(\alpha^{i} p^{i} \nabla \phi, \nabla v\right)=0, & \forall v \in V_{0}, \\
(\epsilon \nabla \phi, \nabla v)-\left(g\left(p^{i}\right), v\right)=\left(\rho^{f}, v\right), & \forall v \in C_{0}^{\infty}(\Omega),
\end{array}
$$

where $\alpha^{i}=D^{i} \beta q^{i}, g\left(p^{i}\right)=\lambda \sum_{i} q^{i} p^{i}, V_{0}=\left\{v\left|v \in H^{1}\left(\Omega_{s}\right), v\right|_{\partial \Omega}=0\right\}$.

Suppose there exists a unique solution $\left(\phi, p^{i}\right)(1 \leq i \leq n)$ satisfying (2.3a) and (2.3b). The finite element approximation to the solution of (2.3a) and (2.3b) is that: find $\phi_{h} \in S_{0}^{h}(\Omega)$ and $p_{h}^{i} \in S^{h}\left(\Omega_{S}\right) \cap V(1 \leq i \leq n)$ such that

$$
\begin{array}{ll}
\left(D^{i} \nabla p_{h}^{i}, \nabla v_{h}\right)+\left(\alpha^{i} p_{h}^{i} \nabla \phi_{h}, \nabla v_{h}\right)=0, & \forall v_{h} \in S_{0}^{h}\left(\Omega_{s}\right) \cap V_{0}, \\
\left(\epsilon \nabla \phi_{h}, \nabla v_{h}\right)-\left(g\left(p_{h}^{i}\right), v_{h}\right)=\sum_{j} q_{j} v_{h}\left(x_{j}\right), & \forall v_{h} \in S_{0}^{h}(\Omega),
\end{array}
$$

where $g\left(p_{h}^{i}\right)=\lambda \sum_{i} q^{i} p_{h}^{i}$. From (2.3b) and (2.4b), we have

$$
\left(\epsilon \nabla\left(\phi-\phi_{h}\right), \nabla v_{h}\right)-\left(g\left(p^{i}-p_{h}^{i}\right), v_{h}\right)=0, \quad \forall v_{h} \in S_{0}^{h}(\Omega) .
$$

Let

$$
a(v, w)=\int_{\Omega} \epsilon \nabla v \nabla w, \quad \forall w, v \in H_{0}^{1}(\Omega) .
$$


Define a Galerkin-projection $R_{h}: H_{0}^{1}(\Omega) \rightarrow S_{0}^{h}(\Omega)$ and a $L^{2}$-projection $Q_{h}: L^{2}(\Omega) \rightarrow S_{0}^{h}(\Omega)$ respectively by

$$
a\left(u-R_{h} u, v\right)=0, \quad \forall v \in S_{0}^{h}(\Omega)
$$

and

$$
\left(u-Q_{h} u, v\right)=0, \quad \forall v \in S_{0}^{h}(\Omega) .
$$

From $[18,23]$, for any $w \in H_{0}^{1}(\Omega) \cap W^{2,2}(\Omega)$, we have the following local error estimates,

$$
\begin{aligned}
& \left\|w-R_{h} w\right\|_{0, \infty, \Omega_{m}} \leq C h^{\frac{1}{2}}|\ln h|\|w\|_{2, \Omega} \\
& \left\|Q_{h} w-R_{h} w\right\|_{0, \infty, \Omega_{m}} \leq C h^{\frac{1}{2}}|\ln h|\|w\|_{2, \Omega}
\end{aligned}
$$

Following [21], we can get a local a priori estimate for the finite element approximation.

Lemma 2.1. Suppose $D \subset \subset \Omega_{0} \subset \subset \Omega$. If $w \in S^{h}\left(\Omega_{0}\right)$ satisfies

$$
a(w, v)=0, \quad \forall v \in S_{h}^{0}\left(\Omega_{0}\right),
$$

then

$$
\|w\|_{1, D} \leq C\|w\|_{0, \Omega_{0}}
$$

Define the Galerkin-projection operator $P_{h}: W_{0}^{1, p}(\Omega)(1 \leq p \leq 3 / 2) \cap H^{1}\left(\Omega_{0}\right) \rightarrow S_{0}^{h}(\Omega)$, satisfying

$$
a\left(\left(\phi-P_{h} \phi\right), v_{h}\right)=\left(g\left(p^{i}-p_{h}^{i}\right), v_{h}\right), \quad \forall v_{h} \in S_{0}^{h}(\Omega) .
$$

Following [21], we can prove the projection operator $P_{h}$ satisfies the following local property.

Lemma 2.2. Let $u \in W_{0}^{1, p}(\Omega) \cap H_{0}^{1}\left(\Omega_{0}\right)$ and $D \subset \subset \Omega_{0}$, then

$$
\left\|P_{h} u\right\|_{1, D} \leq C\left(\|u\|_{1, \Omega_{0}}+\left\|P_{h} u\right\|_{0, \Omega_{0}}+\left\|g\left(p^{i}-p_{h}^{i}\right)\right\|_{0, \Omega_{0}}\right) .
$$

Proof. Let $R_{h}^{0} \equiv P_{h}^{\Omega_{0}}: H_{0}^{1}\left(\Omega_{0}\right) \rightarrow S_{0}^{h}\left(\Omega_{0}\right)$ be the Galerkin projection, i.e., for $w \in H_{0}^{1}\left(\Omega_{0}\right)$,

$$
a\left(w-R_{h}^{0} w, v\right)=\left(g\left(p^{i}-p_{h}^{i}\right), v\right), \quad \forall v \in S_{0}^{h}\left(\Omega_{0}\right) .
$$

Choose $D_{1} \subset \Omega$ satisfying $D \subset \subset D_{1} \subset \subset \Omega_{0}$ and $\omega \in C_{0}^{\infty}\left(\Omega_{0}\right)$ such that $\omega \equiv 1$ on $\bar{D}_{1}$ and supp $\omega \subset \subset \Omega_{0}$. Then for $\tilde{u}=\omega u$,

$$
a\left(R_{h}^{0} \tilde{u}-P_{h} u, v\right)=0, \quad \forall v \in S_{h}^{0}\left(D_{1}\right) .
$$


Thus, Lemma 2.1 yields

$$
\left\|R_{h}^{0} \tilde{u}-P_{h} u\right\|_{1, D} \leq C\left\|R_{h}^{0} \tilde{u}-P_{h} u\right\|_{0, D_{1}} .
$$

On the other hand, from (2.12) and Poincaré inequality, we can easily get the stability for the projection $R_{h}^{0}$

$$
\left\|R_{h}^{0} \tilde{u}\right\|_{1, \Omega_{0}} \leq C\left(\|u\|_{1, \Omega_{0}}+\left\|g\left(p^{i}-p_{h}^{i}\right)\right\|_{0, \Omega_{0}}\right) .
$$

Therefore,

$$
\begin{aligned}
\left\|P_{h} u\right\|_{1, D} & \leq\left\|R_{h}^{0} \tilde{u}\right\|_{1, D}+\left\|R_{h}^{0} \tilde{u}-P_{h} u\right\|_{1, D} \\
& \leq C\left(\left\|R_{h}^{0} \tilde{u}\right\|_{1, D}+\left\|R_{h}^{0} \tilde{u}-P_{h} u\right\|_{0, D_{1}}\right) \\
& \leq C\left(\left\|R_{h}^{0} \tilde{u}\right\|_{1, D_{1}}+\left\|P_{h} u\right\|_{0, D_{1}}\right)
\end{aligned}
$$

which combing with (2.14) completes the proof.

\section{Error estimates for the finite element approximation}

In this section, we first prove some local error bounds for $\phi-\phi_{h}$, then present the error bounds for $p^{i}-p_{h}^{i}$ on the solvent domain $\Omega_{s}$ and $\phi-\phi_{h}$ on the domain $\Omega$. Assume $p^{i} \in$ $H^{1+s}\left(\Omega_{s}\right)(0<s \leq 1)$ and $\phi \in W^{1, \infty}\left(\Omega_{s}\right) \cap H^{1+s}\left(\Omega_{s}\right) \cap W_{0}^{1, p}(\Omega)(0<s \leq 1,1 \leq p<3 / 2)$.

To obtain the local error estimates, we shall make a decomposition of the solution $\phi$ to separate out the singularity caused by the $\delta$ distributions. It is easy to know that $G_{j}=1 /\left(4 \pi \epsilon_{m}\left|x-x_{j}\right|\right)$ is the solution of the following equation

$$
-\epsilon_{m} \Delta G_{j}=\delta\left(x-x_{j}\right), \quad \text { in } \mathbb{R}^{3} .
$$

Let

$$
\tilde{\phi}=\phi-G,
$$

where $G=\sum_{j} q_{j} G_{j}$. Substitute the decomposition (3.2) into the second equation of (2.1), we have

$$
\begin{array}{ll}
-\nabla(\epsilon \nabla \tilde{\phi})-\lambda \sum_{i=1}^{n} q^{i} p^{i}=\nabla\left(\left(\epsilon-\epsilon_{m}\right) \nabla G\right), & \text { in } \Omega, \\
\tilde{\phi}=-G, & \text { on } \partial \Omega .
\end{array}
$$

Thus, the singularities of $\delta$ distribution have been transferred to the function $G$ and the source term $\nabla\left(\left(\epsilon-\epsilon_{m}\right) \nabla G\right) \in H^{-1}(\Omega)$. It is shown in [7] that there is a unique solution $\tilde{\phi} \in H^{1}(\Omega)$, though the original solution $\phi \notin H^{1}(\Omega)$. Since $G \in C^{\infty}(\partial \Omega)$, we can find a function $g \in H^{1}(\Omega)$ such that $g=-G$ on $\partial \Omega$ in the trace sense. We use $V=H_{0}^{1}(\Omega)+g$ to 
denote the affine space with a specified boundary condition and $V_{h}=S^{h}(\Omega) \cap V$ to denote the finite element space of $V$. The weak form of (3.3) is that: Find $\tilde{\phi} \in V$, such that

$$
(\epsilon \nabla \tilde{\phi}, \nabla v)-\left(g\left(p^{i}\right), v\right)=\left(\nabla\left(\left(\epsilon-\epsilon_{m}\right) \nabla G\right), v\right), \quad \forall v \in H_{0}^{1}(\Omega),
$$

where $g\left(p^{i}\right)=\lambda \sum_{i=1}^{n} q^{i} p^{i}$. The finite element approximation to $\tilde{\phi}$ is that: find $\tilde{\phi}_{h} \in V_{h}$, such that

$$
\left(\epsilon \nabla \tilde{\phi}_{h}, \nabla v_{h}\right)-\left(g\left(p_{h}^{i}\right), v_{h}\right)=\left(\nabla\left(\left(\epsilon-\epsilon_{m}\right) \nabla G\right), v_{h}\right), \quad \forall v_{h} \in S^{h}(\Omega) .
$$

Obviously, the function $\tilde{\phi}-\tilde{\phi_{h}}$ satisfies

$$
\left(\epsilon \nabla\left(\tilde{\phi}-\tilde{\phi}_{h}\right), \nabla v_{h}\right)-\left(g\left(p^{i}-p_{h}^{i}\right), v_{h}\right)=0, \quad \forall v_{h} \in S^{h}(\Omega) .
$$

The finite element approximation to the Green function $G$ is that: find $G_{h} \in S^{h}(\Omega)$ such that

$$
\left(\epsilon_{m} \nabla G_{h}, \nabla v_{h}\right)=\sum_{j} q_{j} v_{h}\left(x_{j}\right)
$$

and from (3.1), we have

$$
\left(\epsilon_{m} \nabla\left(G-G_{h}\right), \nabla v_{h}\right)=0, \quad \forall v_{h} \in S^{h}(\Omega) .
$$

We need the following lemmas to present the local error bounds.

Lemma 3.1. If $\phi_{h}, \tilde{\phi}_{h}$ and $G_{h}$ are solutions of (2.4b), (3.5) and (3.7) respectively, we have

$$
\left\|\nabla\left(\phi_{h}-\tilde{\phi}_{h}-G_{h}\right)\right\|_{0, \Omega} \leq C h|\ln h|,
$$

where $C$ depends on $d=\min _{x \in \Omega_{s}} \min _{x_{j}}\left|x-x_{j}\right|$.

Proof. For any $v_{h} \in S_{0}^{h}(\Omega)$, we have

$$
\begin{aligned}
& \left(\epsilon \nabla\left(\phi-\phi_{h}\right), \nabla v_{h}\right)-\left(\epsilon_{m} \nabla\left(G-G_{h}\right), \nabla v_{h}\right)-\left(\epsilon \nabla\left(\tilde{\phi}-\tilde{\phi}_{h}\right), \nabla v_{h}\right) \\
= & \left(\epsilon \nabla\left(\left(\phi-\phi_{h}\right)-\left(G-G_{h}\right)-\left(\tilde{\phi}-\tilde{\phi}_{h}\right)\right), \nabla v_{h}\right)+\left(\left(\epsilon-\epsilon_{m}\right) \nabla\left(G-G_{h}\right), \nabla v_{h}\right) \\
= & -\left(\epsilon \nabla\left(\phi_{h}-\tilde{\phi}_{h}-G_{h}\right), \nabla v_{h}\right)+\left(\left(\epsilon-\epsilon_{m}\right) \nabla\left(G-G_{h}\right), \nabla v_{h}\right) .
\end{aligned}
$$

Thus, from (2.5), (3.6) and (3.8), we get

$$
\left(\epsilon \nabla\left(\phi_{h}-\tilde{\phi}_{h}-G_{h}\right), \nabla v_{h}\right)=\left(\left(\epsilon-\epsilon_{m}\right) \nabla\left(G-G_{h}\right), \nabla v_{h}\right) .
$$

Therefore

$$
\begin{aligned}
\left|\left(\nabla\left(\phi_{h}-\tilde{\phi}_{h}-G_{h}\right), \nabla v_{h}\right)\right| & \leq C\left|\left(\epsilon \nabla\left(\phi_{h}-\tilde{\phi}_{h}-G_{h}\right), \nabla v_{h}\right)\right| \\
& =C\left|\left(\left(\epsilon-\epsilon_{m}\right) \nabla\left(G-G_{h}\right), \nabla v_{h}\right)\right| \\
& \leq C\left\|\nabla\left(G-G_{h}\right)\right\|_{0, \Omega_{s}}\left\|\nabla v_{h}\right\|_{0, \Omega} .
\end{aligned}
$$


By using the local estimate (cf. [26])

$$
\left\|\nabla\left(G-G_{h}\right)\right\|_{0, \infty, \Omega_{s}} \leq C h,
$$

we have

$$
\left\|\nabla\left(\phi_{h}-\tilde{\phi}_{h}-G_{h}\right)\right\|_{0, \Omega} \leq C h,
$$

where $C$ depends on $d=\min _{x \in \Omega_{s}} \min _{x_{j}}\left|x-x_{j}\right|$. This completes the proof of the lemma.

The following $L^{2}$ norm error estimate is obtained by the standard duality argument. Lemma 3.2. If $\phi_{h}, \tilde{\phi}_{h}$ and $G_{h}$ are solutions of (2.4b), (3.5) and (3.7) respectively, we have

$$
\left\|\phi_{h}-\tilde{\phi}_{h}-G_{h}\right\|_{0, \Omega} \leq C h^{\frac{3}{2}}|\ln h|^{\frac{3}{2}},
$$

where $C$ depends on $d=\min _{x \in \Omega_{s}} \min _{x_{j}}\left|x-x_{j}\right|$.

Proof. Let $w \in H_{0}^{1}(\Omega)$ be the solution of the following auxiliary problem: find $w \in H_{0}^{1}(\Omega)$ satisfying

$$
(\epsilon \nabla w, \nabla v)=\left(\phi_{h}-\tilde{\phi}_{h}-G_{h}, v\right), \quad \forall v \in H_{0}^{1}(\Omega) .
$$

The finite element approximation to $w$ is that: find $w_{h} \in S_{0}^{h}(\Omega)$ satisfying

$$
\left(\epsilon \nabla w_{h}, \nabla v_{h}\right)=\left(\phi_{h}-\tilde{\phi}_{h}-G_{h}, v_{h}\right), \quad \forall v_{h} \in S_{0}^{h}(\Omega) .
$$

We know that $w \in X=H_{0}^{1}(\Omega) \cap H^{2}\left(\Omega_{s}\right) \cap H^{2}\left(\Omega_{m}\right)$ satisfying (cf. [8])

$$
\|w\|_{X} \leq C\left\|\phi_{h}-\tilde{\phi}_{h}-G_{h}\right\|_{0, \Omega},
$$

where the norm $\|w\|_{X}=\|w\|_{1, \Omega}+\|w\|_{2, \Omega_{s}}+\|w\|_{2, \Omega_{m}}$ and

$$
\left\|\nabla\left(w-w_{h}\right)\right\|_{0, \Omega} \leq C h|\ln h|^{\frac{1}{2}}\left\|\phi_{h}-\tilde{\phi}_{h}-G_{h}\right\|_{0, \Omega} .
$$

Taking $v=\phi_{h}-\tilde{\phi}_{h}-G_{h}$ in (3.13), we have

$$
\begin{aligned}
\left\|\phi_{h}-\tilde{\phi}_{h}-G_{h}\right\|_{0, \Omega}^{2} & =\left(\epsilon \nabla w, \nabla\left(\phi_{h}-\tilde{\phi}_{h}-G_{h}\right)\right) \\
& =\left(\epsilon \nabla\left(w-w_{h}\right), \nabla\left(\phi_{h}-\tilde{\phi}_{h}-G_{h}\right)\right)+\left(\left(\epsilon \nabla w_{h}, \nabla\left(\phi_{h}-\tilde{\phi}_{h}-G_{h}\right)\right)\right. \\
& =(I)_{1}+(I)_{2} .
\end{aligned}
$$

We find from Lemma 3.1 and (3.14) that

$$
\begin{aligned}
(I)_{1} & \leq C\left\|\nabla\left(w-w_{h}\right)\right\|_{0, \Omega}\left\|\nabla\left(\phi_{h}-\tilde{\phi}_{h}-G_{h}\right)\right\|_{0, \Omega} \\
& \leq C h^{2}|\ln h|^{\frac{3}{2}}\left\|\phi_{h}-\tilde{\phi}_{h}-G_{h}\right\|_{0, \Omega} .
\end{aligned}
$$


From (3.10), we get

$$
\begin{aligned}
(I)_{2} & =\left(\left(\epsilon-\epsilon_{m}\right) \nabla w_{h}, \nabla\left(G-G_{h}\right)\right) \\
& =\left(\left(\epsilon-\epsilon_{m}\right) \nabla\left(w_{h}-w\right), \nabla\left(G-G_{h}\right)\right)+\left(\left(\epsilon-\epsilon_{m}\right) \nabla w, \nabla\left(G-G_{h}\right)\right) \\
& \leq C\left\|\nabla\left(w_{h}-w\right)\right\|_{0, \Omega}\left\|\nabla\left(G_{h}-G\right)\right\|_{0, \Omega_{s}}+\|w\|_{X}\left\|G_{h}-G\right\|_{0, \Omega_{s}}+\|w\|_{1, \Omega}\left\|G_{h}-G\right\|_{0, \lambda \Omega_{s}} \\
& \leq C h^{\frac{3}{2}}|\ln h|^{\frac{1}{2}}\left\|\phi_{h}-\tilde{\phi}_{h}-G_{h}\right\|_{0, \Omega} .
\end{aligned}
$$

Thus, we complete the proof of this lemma by combining (3.15), (3.16) and (3.17).

Now we can present the local error bounds in $L^{2}$ norm and $H^{1}$ norm respectively for $\phi-\phi_{h}$.

Theorem 3.1. Suppose domain $\Omega_{1} \subset \subset \Omega$ and all the singular points $x_{j} \notin \Omega_{1}$. The following error bound holds

$$
\left\|\phi-\phi_{h}\right\|_{0, \Omega_{1}} \leq C\left(h^{\frac{3}{2}}|\ln h|^{\frac{3}{2}}+\left\|g\left(p^{i}-p_{h}^{i}\right)\right\|_{0, \Omega_{s}}\right),
$$

when $h$ is sufficiently small and $C$ depends on $d=\min _{x \in \Omega_{1}} \min _{x_{j}}\left|x-x_{j}\right|$.

Proof. First, we have

$$
\left\|\phi-\phi_{h}\right\|_{0, \Omega_{1}} \leq\left\|\tilde{\phi}-\tilde{\phi}_{h}\right\|_{0, \Omega_{1}}+\left\|G-G_{h}\right\|_{0, \Omega_{1}}+\left\|\tilde{\phi}_{h}+G_{h}-\phi_{h}\right\|_{0, \Omega_{1}} .
$$

We find from [18] that

$$
\left\|G-G_{h}\right\|_{0, \Omega_{1}} \leq\left\|G-G_{h}\right\|_{0, \infty, \Omega_{1}} \leq C h^{2}|\ln h|^{2},
$$

where $C$ depends on $d=\min _{x \in \Omega_{1}} \min _{x_{j}}\left|x-x_{j}\right|$.

By using the same argument in [4], it is easy to obtain

$$
\left\|\tilde{\phi}-\tilde{\phi}_{h}\right\|_{0, \Omega} \leq C\left(\left\|g\left(p^{i}-p_{h}^{i}\right)\right\|_{0, \Omega_{s}}+h^{2}|\ln h|\left\|\nabla\left(\epsilon-\epsilon_{m}\right) \nabla G\right\|_{0, \Omega}\right) .
$$

From [18], we obtain

$$
\left\|\nabla\left(\epsilon-\epsilon_{m}\right) \nabla G\right\|_{0, \Omega} \leq\left\|\nabla\left(\epsilon_{s}-\epsilon_{m}\right) \nabla G\right\|_{0, \infty, \Omega_{s}} \leq C,
$$

where $C$ depends on $d$ too. Hence,

$$
\left\|\tilde{\phi}-\tilde{\phi}_{h}\right\|_{0, \Omega} \leq C\left(h^{2}|\ln h|+\left\|g\left(p^{i}-p_{h}^{i}\right)\right\|_{0, \Omega_{s}}\right) .
$$

Now, we complete the proof from Lemma 3.2, (3.19), (3.20) and (3.21).

Theorem 3.2. For $\phi \in H^{1+s} \cap W_{0}^{1, p}(\Omega)(0<s \leq 1,1 \leq p<3 / 2)$, the following error bound holds

$$
\left\|\phi-\phi_{h}\right\|_{1, \Omega_{s}} \leq C\left(h^{s}+\left\|g\left(p^{i}-p_{h}^{i}\right)\right\|_{0, \Omega_{0}}\right) .
$$


Proof. From Lemma 2.2, for $v \in S_{0}^{h}\left(\Omega_{0}\right)$ we have

$$
\begin{aligned}
\left\|\phi-P_{h} \phi\right\|_{1, \Omega_{s}} & \leq\|\phi-v\|_{1, \Omega_{s}}+\left\|P_{h}(\phi-v)\right\|_{1, \Omega_{s}} \\
& \leq\|\phi-v\|_{1, \Omega_{0}}+\left\|P_{h}(\phi-v)\right\|_{0, \Omega_{0}}+\left\|g\left(p^{i}-p^{i}\right)\right\|_{0, \Omega_{0}} \\
& \leq C\left(\inf _{v \in S_{0}^{h}\left(\Omega_{0}\right)}\|\phi-v\|_{1, \Omega_{0}}+\left\|P_{h} \phi-\phi\right\|_{0, \Omega_{0}}+\left\|g\left(p^{i}-p_{h}^{i}\right)\right\|_{0, \Omega_{0}}\right),
\end{aligned}
$$

where $\Omega_{s} \subset \Omega_{0}$ and $\Omega_{0}$ do not include the singular points $x_{j}, j=1, \cdots, N_{m}$. We complete the proof of this theorem from Theorem 3.1.

Now, we can present the error bounds for $p^{i}-p_{h}^{i}$.

Theorem 3.3. Let $p^{i} \in H^{1+s}\left(\Omega_{s}\right)$ and $p_{h}^{i}$ be the solution of (2.3a) and (2.4a) $(0<s \leq 1)$, respectively, for any $v_{h} \in S_{0}^{h}\left(\Omega_{s}\right) \cap V_{0}$, we have

$$
\begin{gathered}
\left\|p^{i}-p_{h}^{i}\right\|_{1, \Omega_{s}} \leq C\left(\left(h^{s}+\left\|g\left(p^{i}-p_{h}^{i}\right)\right\|_{0, \Omega_{s}}\right)\left(1+h^{-\frac{3}{2}}\left\|p^{i}-p_{h}^{i}\right\|_{0, \Omega_{s}}\right)\right. \\
\left.+\left\|p^{i}-v_{h}\right\|_{1, \Omega_{s}}\right), \quad \frac{1}{2}<s \leq 1
\end{gathered}
$$

and if $p_{h}^{i} \in L^{\infty}\left(\Omega_{s}\right)$, then

$$
\left\|p^{i}-p_{h}^{i}\right\|_{1, \Omega_{s}} \leq C\left(h^{s}+\left\|g\left(p^{i}-p_{h}^{i}\right)\right\|_{0, \Omega_{s}}+\left\|p^{i}-v_{h}\right\|_{1, \Omega_{s}}\right), \quad 0<s \leq 1 .
$$

Proof. First, we give the proof for (3.24). Set $e=p^{i}-p_{h}^{i}$. By the definition of $p^{i}, p_{h}^{i}$, the error $e$ satisfies

$$
\left(D^{i} \nabla e, \nabla w_{h}\right)+\left(\alpha^{i}\left(p^{i} \nabla \phi-p_{h}^{i} \nabla \phi_{h}\right), \nabla w_{h}\right)=0, \quad \forall w_{h} \in S_{0}^{h}\left(\Omega_{s}\right) \cap V_{0} .
$$

For any $v_{h} \in S_{0}^{h}\left(\Omega_{s}\right) \cap V_{0}$,

$$
\|\nabla e\|_{0, \Omega_{s}}^{2} \leq\left(D^{i} \nabla e, \nabla e\right)=\left(D^{i} \nabla e, \nabla\left(p^{i}-v_{h}\right)\right)+\left(D^{i} \nabla e, \nabla\left(v_{h}-p_{h}^{i}\right)\right) .
$$

Hence, from (3.25), we get

$$
\begin{aligned}
\|\nabla e\|_{0, \Omega_{s}}^{2} \leq & \|\nabla e\|_{0, \Omega_{s}}\left\|p^{i}-v_{h}\right\|_{1, \Omega_{s}}-\left(\alpha^{i}\left(p^{i} \nabla \phi-p_{h}^{i} \nabla \phi_{h}\right), \nabla\left(v_{h}-p_{h}^{i}\right)\right) \\
= & \|\nabla e\|_{0, \Omega_{s}}\left\|p^{i}-v_{h}\right\|_{1, \Omega_{s}}+\left(\alpha^{i}\left(p^{i} \nabla \phi-p_{h}^{i} \nabla \phi_{h}\right), \nabla\left(p^{i}-v_{h}\right)\right) \\
& -\left(\alpha^{i}\left(p^{i} \nabla \phi-p_{h}^{i} \nabla \phi_{h}\right), \nabla e\right) .
\end{aligned}
$$

If $\left\|p_{h}^{i}\right\|_{0, \infty} \leq C$, then we have

$$
\begin{aligned}
\left(\alpha^{i}\left(p^{i} \nabla \phi-p_{h}^{i} \nabla \phi_{h}\right), \nabla\left(p^{i}-v_{h}\right)\right) & =\left(\alpha^{i}\left(p^{i}-p_{h}^{i}\right) \nabla \phi+\alpha^{i} p_{h}^{i}\left(\nabla \phi-\nabla \phi_{h}\right), \nabla\left(p^{i}-v_{h}\right)\right) \\
& \leq C\left(\left\|p^{i}-p_{h}^{i}\right\|_{0, \Omega_{s}}+\left\|\nabla \phi-\nabla \phi_{h}\right\|_{0, \Omega_{s}}\right)\left\|\nabla\left(p^{i}-v_{h}\right)\right\|_{0, \Omega_{s}} \\
& \leq C\left(h^{s}+\left\|g\left(p^{i}-p_{h}^{i}\right)\right\|_{0, \Omega_{s}}\right)\left\|\nabla\left(p^{i}-v_{h}\right)\right\|_{0, \Omega_{s}}
\end{aligned}
$$


in which we have used Theorem 3.2. Similarly, we can prove

$$
\left(\alpha^{i}\left(p^{i} \nabla \phi-p_{h}^{i} \nabla \phi_{h}\right), \nabla\left(p^{i}-p_{h}^{i}\right)\right) \leq C\left(h^{s}+\left\|g\left(p^{i}-p_{h}^{i}\right)\right\|_{0, \Omega_{s}}\right)\|\nabla e\|_{0, \Omega_{s}} .
$$

Hence, from (3.27), (3.28) and (3.29), we have

$$
\|\nabla e\|_{0, \Omega_{s}}^{2} \leq C\left(h^{2 s}+\left\|p^{i}-v_{h}\right\|_{1, \Omega_{s}}^{2}+\left\|g\left(p^{i}-p_{h}^{i}\right)\right\|_{0, \Omega_{s}}^{2}\right) .
$$

This completes the proof of (3.24). Second, to avoid using maximum bound for $p_{h}^{i}$, the second term on the right side in (3.26) is estimated by

$$
\begin{aligned}
\left(D^{i} \nabla e, \nabla\left(v_{h}-p_{h}^{i}\right)\right)= & -\left(\alpha^{i}\left(p^{i} \nabla \phi-p_{h}^{i} \nabla \phi_{h}\right), \nabla\left(v_{h}-p_{h}^{i}\right)\right) \\
=- & \left(\alpha^{i}\left(\left(\nabla \phi-\nabla \phi_{h}\right)\left(p^{i}-e\right)+\nabla \phi e\right), \nabla\left(v_{h}-p_{h}^{i}\right)\right) \\
\leq & C\left(\left\|\nabla \phi-\nabla \phi_{h}\right\|_{0, \Omega_{s}}\left(\left\|\nabla\left(v_{h}-p_{h}^{i}\right)\right\|_{0, \Omega_{s}}+\|e\|_{0,3}\left\|\nabla\left(v_{h}-p_{h}^{i}\right)\right\|_{0,6}\right)\right. \\
& \left.\quad+\|e\|_{0, \Omega_{s}}\left\|\nabla\left(v_{h}-p_{h}^{i}\right)\right\|_{0, \Omega_{s}}\right),
\end{aligned}
$$

in which we have used (3.25). By interpolation error estimate and an inverse estimate, we have

$$
\begin{aligned}
\|e\|_{0,3} & \leq\left\|p_{h}^{i}-\Pi_{h} p^{i}\right\|_{0,3}+\left\|\Pi_{h} p^{i}-p^{i}\right\|_{0,3} \\
& \leq C\left(h^{-\frac{1}{2}}\left\|p_{h}^{i}-\Pi_{h} p^{i}\right\|_{0, \Omega_{s}}+h^{s+\frac{1}{2}}\left\|p^{i}\right\|_{1+s, 2}\right) \\
& \leq C\left(h^{-\frac{1}{2}}\|e\|_{0, \Omega_{s}}+h^{s+\frac{1}{2}}\right),
\end{aligned}
$$

and

$$
\left\|\nabla\left(v_{h}-p_{h}^{i}\right)\right\|_{0,6} \leq C h^{-1}\left\|\nabla\left(v_{h}-p_{h}^{i}\right)\right\|_{0, \Omega_{s}} .
$$

Hence, Eq. (3.31) becomes

$$
\begin{aligned}
\left(D^{i} \nabla e, \nabla\left(v_{h}-p_{h}^{i}\right)\right) & \leq C\left(1+h^{-\frac{3}{2}}\|e\|_{0, \Omega_{s}}\right)\left(h^{s}+\left\|g\left(p^{i}-p_{h}^{i}\right)\right\|_{0, \Omega_{s}}\right)\left\|\nabla\left(v_{h}-p_{h}^{i}\right)\right\|_{0, \Omega_{s}} \\
& \leq C\left(1+h^{-\frac{3}{2}}\|e\|_{0, \Omega_{s}}\right)^{2}\left(h^{s}+\left\|g\left(p^{i}-p_{h}^{i}\right)\right\|_{0, \Omega_{s}}\right)^{2}+C_{0}\left\|\nabla\left(v_{h}-p_{h}^{i}\right)\right\|_{0, \Omega_{s}}^{2}
\end{aligned}
$$

where $C_{0}<1$ is a constant independent of $h$. Hence,

$$
\|\nabla e\|_{1, \Omega_{s}}^{2} \leq C\left\|p^{i}-v_{h}\right\|_{1, \Omega_{s}}^{2}+C\left(1+h^{-\frac{3}{2}}\|e\|_{0, \Omega_{s}}\right)^{2}\left(h^{s}+\left\|g\left(p^{i}-p_{h}^{i}\right)\right\|_{0, \Omega_{s}}\right)^{2} .
$$

We complete the proof of (3.23). This completes the proof of Theorem 3.3.

At last, by using (2.8a), we can obtain the following error bound for $\phi-\phi_{h}$ on the domain $\Omega$. 
Theorem 3.4. There holds

$$
\left\|\phi-\phi_{h}\right\|_{0, \Omega} \leq C\left((1+|\ln h|) h^{\frac{1}{2}}+\left\|g\left(p^{i}-p_{h}^{i}\right)\right\|_{0, \Omega_{s}}\right) .
$$

Proof. Following [23], for any $f \in L^{2}(\Omega)$, there exists $w \in H_{0}^{1}(\Omega) \cap H^{2}(\Omega)$ such that

$$
\left\{\begin{array}{l}
a(w, v)=(f, v), \quad \\
\|w\|_{2, \Omega} \leq C\|f\|_{0, \Omega},
\end{array} \quad \forall v \in H_{0}^{1}(\Omega),\right.
$$

where $a(\cdot, \cdot)$ is defined by (2.6). Hence, from (2.5), (2.3b) and (2.8a), we have

$$
\begin{aligned}
\left|\left(\phi-\phi_{h}, f\right)\right|= & \left|a\left(\phi-\phi_{h}, w\right)\right| \\
\leq & \left|a\left(\phi, w-R_{h}^{*} w\right)\right|+\left|\left(g\left(p^{i}-p_{h}^{i}\right), R_{h}^{*} w\right)\right| \\
\leq & C\left(\left\|w-R_{h}^{*} w\right\|_{0, \infty, \Omega_{m}}+\left\|g\left(p^{i}\right)\right\|_{0, \Omega_{s}}\left\|w-R_{h}^{*} w\right\|_{0, \Omega_{s}}\right) \\
& \quad+C\left(\left\|g\left(p^{i}-p_{h}^{i}\right)\right\|_{0, \Omega_{s}}\left\|R_{h}^{*} w\right\|_{0, \Omega_{s}}\right) \\
\leq & C\left((1+|\ln h|) h^{\frac{1}{2}}+\left\|g\left(p^{i}-p_{h}^{i}\right)\right\|_{0, \Omega_{s}}\right)\|w\|_{2, \Omega},
\end{aligned}
$$

which together with (3.35) obtains (3.34). This completes the proof.

\section{Numerical experiments}

In this section, we will report two numerical experiments including a biomolecular problem to illustrate the theoretical results obtained in this paper.

Our numerical experiments were carried out on LSSC-II in the State Key Laboratory of Scientific and Engineering Computing, Chinese Academy of Sciences.

Since the surface of the molecule such as protein or DNA is extremely irregular in practice, the initial mesh for the computation of PNP equations should be nonuniform with a fine mesh around the surface and a coarse mesh away from the surface. Thus in the following experiments we illustrate the convergence rate in terms of the order of the degree of freedoms $N$ instead of that of the mesh size $h$. For example, if $\phi \in H^{2}\left(\Omega_{s}\right)$ and $\left\|p^{i}-p_{h}^{i}\right\|_{0, \Omega_{s}}=\mathcal{O}(h), i=1, \cdots, n$, then the theoretical result for the $H^{1}$ error estimate for the electrostatic potential is $\left\|\phi-\phi_{h}\right\|_{1, \Omega_{s}}=\mathcal{O}(h)$ (see (3.22)). In our experiments, we think the experiment result is consistent with the theoretical one if it satisfies $\left\|\phi-\phi_{h}\right\|_{1, \Omega_{s}}=$ $\mathcal{O}\left(N^{-1 / 3}\right)$. In addition, since there is no analytic solution for the standard PNP system with discontinuous coefficients, we use the computational solution on a very fine mesh instead of the analytic one as a reference solution.

Example 4.1. The first example is to solve the steady-state PNP equations (2.1) for two species of particles, one carries charge -1 and the other +1 , in the spherical annulus $\Omega_{s}$ between $r=1 \AA$ and $r=200 \AA$ with a constant diffusion coefficient $D^{i}$, a value $\beta=1.6774$ and ion densities $p_{\text {pulk }}^{i}=50 \mathrm{mM}, i=1,2$ under the boundary condition (2.2). 


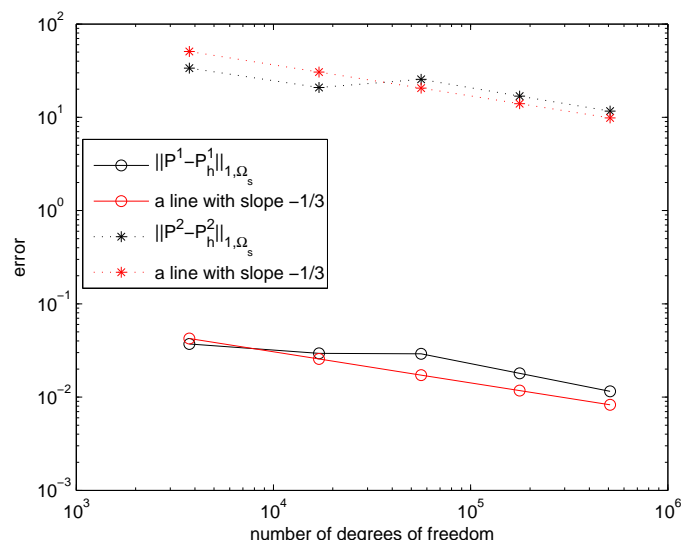

Figure 2: The convergence curves of $\left\|p^{i}-p_{h}^{i}\right\|_{1, \Omega_{s}}, i=1,2$ for Example 4.1.

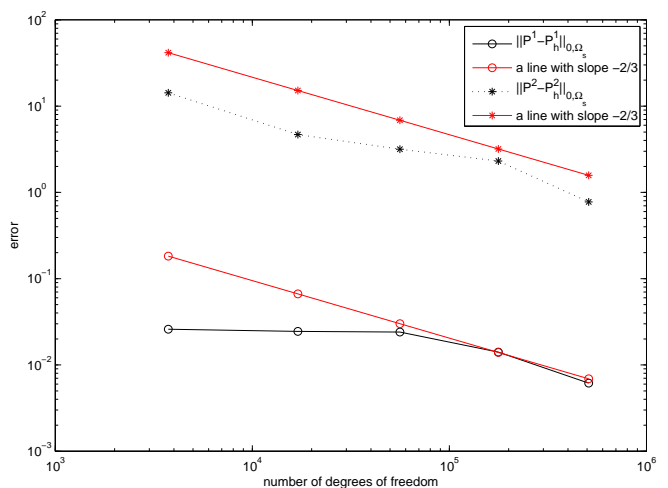

Figure 3: The convergence curves of $\left\|p^{i}-p_{h}^{i}\right\|_{0, \Omega_{s}}, i=1,2$ for Example 4.1.

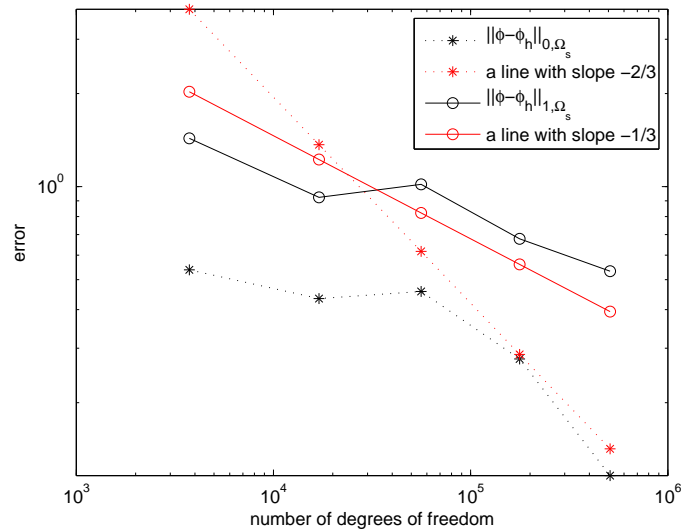

Figure 4: The convergence curves of $\left\|\phi-\phi_{h}\right\|_{1, \Omega_{s}}$ and $\left\|\phi-\phi_{h}\right\|_{0, \Omega_{s}}$ for Example 4.1. 
The initial mesh is generated through a procedure as described in [14] and [15]. Our numerical results are presented in Figs. 2, 3 and 4. It is shown from Figs. 2 and 3 that the convergence curves of $H^{1}$ and $L^{2}$ errors for the densities of two species approximate to the lines with slope $-1 / 3$ and $-2 / 3$, respectively. These mean that the finite element approximations to the densities have the optimal convergence rates for the spherical case, which coincides with our theory in Section 3 (If $\left\|p^{i}-p_{h}^{i}\right\|_{0, \Omega_{s}}=\mathcal{O}\left(h^{2}\right)$, from (3.24), $\| p^{i}-$ $\left.p_{h}^{i} \|_{1, \Omega_{s}}=\mathcal{O}(h)\right)$. The similar conclusion can be obtained for the electrostatic potential $\phi$ from Fig. 4.

Example 4.2. The second example is to solve (2.1) for a diffusion-reaction process of neurotransmitter acetylcholine (ACh) at the reaction center of the enzyme acetylcholinesterase (AChE) (cf. [14]). The ACh molecules are treated as particles with +1 charge and the computation domain is chosen to be a ball with a radius $400 \AA$ centered at the geometric center of the AChE molecule. We consider two other species of nonreactive particles, one with +1 charge and the other with -1 charge. The boundary condition and the interface conditions for the electrostatic potential are shown in (2.2). Denote the flux across the interface for the $i$-th species by

$$
J_{i}=n \cdot D^{i}\left(\nabla p^{i}+\beta q^{i} p^{i} \nabla \phi\right) .
$$

The boundary conditions for these two species of non-reactive particles are the same as in (2.2)

$$
\begin{cases}J_{i}=0, & \text { on } \Gamma, \quad 1 \leq i \leq 2, \\ p^{i}=p_{p u l k}^{i}, & \text { on } \partial \Omega, \quad 1 \leq i \leq 2 .\end{cases}
$$

The boundary conditions for ACh are defined as follows:

$$
\begin{cases}p^{A C h}=0, & \text { on } \Gamma_{a}, \\ J_{A C h}=0, & \text { on } \Gamma \backslash \Gamma_{a}, \\ p^{A C h}=p_{p u l k}^{A C h}, & \text { on } \partial \Omega .\end{cases}
$$

The bulk concentration of all three species of particles are set to be $50 \mathrm{mM}$.

The numerical results for this example are presented in Figs. 5, 6 and 7. It is shown in Fig. 5 that the $L^{2}$ norm error $\left\|p^{i}-p_{h}^{i}\right\|_{0, \Omega_{s}}$ approximates $\mathcal{O}\left(h^{2}\right)$ and in Fig. 6 that the $H^{1}$ norm error $\left\|p^{i}-p_{h}^{i}\right\|_{1, \Omega_{s}}$ approximates $\mathcal{O}(h)$, which supports our theory in Section 3 . The similar conclusion can be obtained for the electrostatic potential $\phi$ from Fig. 7.

\section{Acknowledgments}

Deep thanks must be expressed to Professor Aihui Zhou for his helpful discussions and valuable suggestions. Thanks also go to $X$. Xie and B. Tu for implementing the numerical experiments. Y. Yang was supported by the China NSF (NSFC 11001062, NSFC 


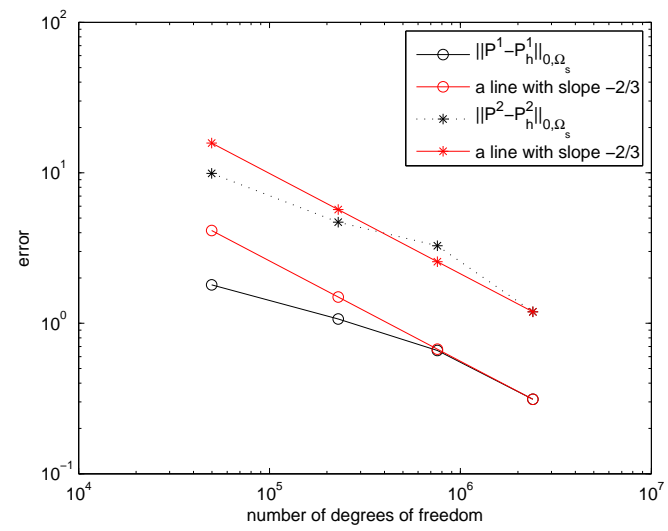

Figure 5: The convergence curves of $\left\|p^{i}-p_{h}^{i}\right\|_{0, \Omega_{s}}, i=1,2$ for Example 4.2.

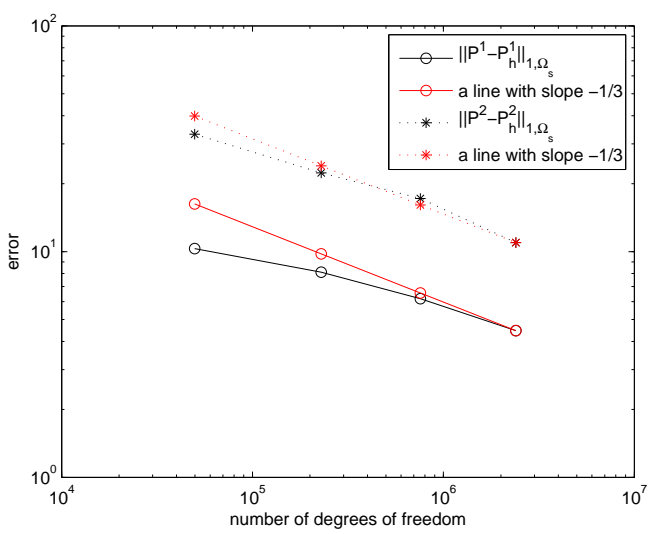

Figure 6: The convergence curves of $\left\|p^{i}-p_{h}^{i}\right\|_{1, \Omega_{s}}, i=1,2$ for Example 4.2.

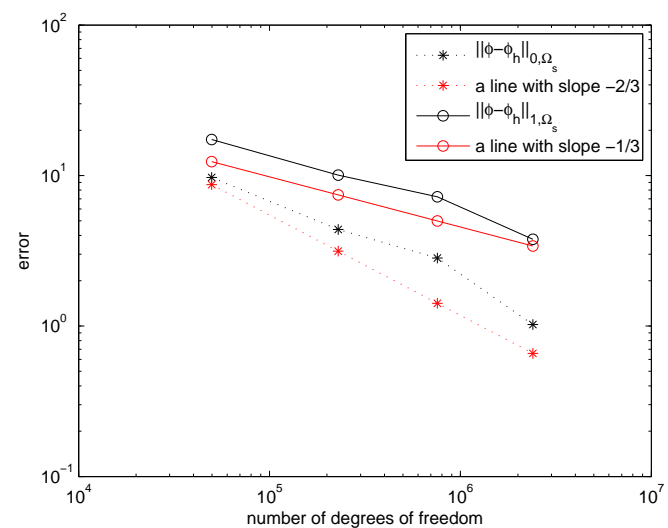

Figure 7: The convergence curves of $\left\|\phi-\phi_{h}\right\|_{1, \Omega_{s}}$ and $\left\|\phi-\phi_{h}\right\|_{0, \Omega_{s}}$ for Example 4.2. 
11161014) and the fund from Education Department of Guangxi Province under grant 201012MS094. B. Z. Lu was supported by the National Center for Mathematics and Interdisciplinary Sciences, Chinese Academy of Sciences and the China NSF (NSFC10971218).

\section{References}

[1] R. A. AdAms, Sobolev Spaces, Academic Press, New York, 1975.

[2] W. AllegretTO, Y. LiN AND A. ZHOU, A box scheme for coupled systems resulting from microsensor thermistor problems, Dynam. Contin. Discrete Impuls. Systems, 5 (1999), pp. 209-223.

[3] S. C. BRENNER AND L. R. SCOTT, The Mathematical Theory of Finite Element Methods, Springer-Verlag, New York, 1994.

[4] I. BABUŠKA, The finite element method for elliptic equations with discontinuous coefficients, Computing, 5 (1970), pp. 207-213.

[5] V. BARCILON, D. P. Chen, R. S. EISEnberG AND J. W. Jerome, Qualitative properties of steady-state Poisson-Nernst-Planck systems: perturbation and simulation study, SIAM J. Appl. Math., 3 (1997), pp. 631-648.

[6] A. E. Cardenas, R. D. Coalson and M. G. Kurnikova, Three-dimensional PoissonNernst-Planck theory studies: influence of membrane electrostatics on gramicidin a channel conductance, Biophys. J., 79(1) (2000), pp. 80-93.

[7] L. Chen, M. J. HOLST AND J. XU, The finite element approximation of the nonlinear PoissonBoltzmann equation, SIAM J. Numer. Anal., 45 (2007), pp. 2298-2320.

[8] Z. CHEN AND J. ZHEN, Finite element methods and their convergence for elliptic and parabolic interface problem, Numer. Math., 79 (1998), pp. 175-202.

[9] D. S. Bolintineanu, A. Sayyed-Ahmad, H. T. Davis and Y. N. Kaznessis, PoissonNernst-Planck models of nonequilibrium ion electrodiffusion through a protegrin transmembrane pore, PLoS Comput. Biol., 5 (2009), e1000277.

[10] R. Eisenberg AND D. P. Chen, Poisson-Nernst-Planck (PNP) theory of an open ionic channel, Biophys. J., 64(2) (1993), A22-A22.

[11] C. M. ElLIOTT AND S. LARSSON, A finite element model for the time-dependent joule heating problem, Math. Comput., 64 (1995), pp. 1433-1453.

[12] J. W. JEROME, Consistency of semiconductor modeling: an existence/ stability analysis for the stationary van Boosbroeck system, SIAM J. Appl. Math., 45 (1985), pp. 565-590.

[13] M. G. KuRNiKova, R. D. COALSON, P. GRAF AND A. NitZAN, A lattice relaxation algorithm for three-dimensional Poisson-Nernst-Planck theory with application to ion transpoort through the gramicidin a channel, Biophys. J., 76(2) (1999), pp. 642-656.

[14] B. Z. LU, M. J. Holst, J. A. MCCAmmo And Y. C. ZHOU, Poisson-Nernst-Planck equations for simulating biomolecular diffusion-reaction processes I: finite element solutions, J. Comput. Phys., 229 (2010), pp. 6979-6994.

[15] B. Z. LU, Y. C. ZhOu, G. A. Huber, S. D. Bond, M. J. Holst and J. A. McCammon, Electrodiffusion: a continuum modeling framework for biomolecular systems with realistic spatiotemporal resolution, J. Chem. Phys., 127 (2007), 135102.

[16] W. NERNST, Die elektromotorische wirksamkeit der ionen, Z. Physik. Chem., 4 (1889), pp. 4-129.

[17] M. PlanCK, Über die erregung von electricität und wärme in electrolyten, Ann. Phys. Chem., (1890), pp. 39-161.

[18] A. H. SCHATZ AND L. B. WAHLBIN, Interior maximum norm estimates for finite element methods, Math. Comput., 31 (1977), pp. 414-442. 
[19] Y. H. SONG, Y. J. ZHANG, C. L. BAJAJ AND N. A. BAKER, Continuum diffusion reaction rate calculations of wild-type and mutant mouse acetylcholinesterase: adaptive finite element analysis, Biophys. J., 3 (2004), pp. 1558-1566.

[20] Y. H. Song, Y. J. Zhang, T. Y. Shen, C. L. BAJAJ, J. A. MCCAMmon And N. A. BAKer, Finite element solution of the steady-state Smoluchowski equation for rate constant calculations, Biophys. J., 4 (2004), pp. 2017-2029.

[21] J. XU AND A. ZHOU, Local and parallel finite element algprithms based on two-grid discretizations, Math. Comput., 69 (2000), pp. 881-909.

[22] Y. YANG AND A. ZHOU, A finite element recovery approach to Green's function approximations with applications to electrostatic potential computation, J. Comput. Appl. Math., 225 (2009), pp. 202-212.

[23] Y. YANG AND A. ZHOU, Two-scale finite element Green's function approximations with applications to electrostatic potential computation, J. Syst. Sci. Complex., 23 (2010), pp. 177-193.

[24] X. YUE, Numerical analysis of nonstationary thermistor problem, J. Comput. Math., 12 (1994), pp. 213-223.

[25] A. ZHOU, C. LIEM, T. SHIH AND L. LÜ, Error analysis on bi-parameter finite element, Comput. Methods Appl. Mech. Eng., 158 (1998), pp. 329-339.

[26] Q. ZHU AND Q. LIN, Superconvergence Theory of Finite Element Methods, Hunan Science Press, Changsha, 1989 (in Chinese).

[27] Y. C. Zhou, B. Z. Lu, G. A. Huber, M. J. Holst And J. A. McCammon, Continuum simulations of acetylcholine consumption by acetylcholinesterase-a Poisson-Nernst-Planck approach, J. Phys. Chem. B, 112(2) (2008), pp. 270-275.

[28] Z. ZHOU, P. PAYNE, M. VASQUEZ, N. KUHN AND M. LEVITT, Finite-difference solution of the Poisson-Boltzmann equaton: complete elimination of self-energy, J. Comput. Chem., 11 (1996), pp. 1344-1351. 